\title{
Voluntary euthanasia is now legal in Quebec, says appeal court
}

In this News story (BMJ 2015;351:h7009, doi:10.1136/bmj. h7009), we incorrectly stated that "Quebec will allow assisted suicide, in which a doctor prescribes a lethal drug for a patient to take, and voluntary euthanasia, in which a physician directly administers the lethal dose on request." In fact, only voluntary euthanasia will be permitted in the province. We apologise for this error

Cite this as: BMJ 2016;352:171

๑ BMJ Publishing Group Ltd 2016 